\title{
Sudden unexpected death in infancy (SUDI)
}

\author{
C M Loughrey, M A Preece, A Green
}

\section{Rigorous metabolic investigation is essential}

$\mathrm{S}$ udden infant death syndrome (SIDS) risk reduction campaigns have resulted in a significant decline in the incidence of SIDS. ${ }^{1}$ However, SIDS remains the major single cause of death in children in developed countries, with a reported incidence, together with unascertained deaths, of approximately $1 / 3000$ live births. ${ }^{12}$

SIDS is in essence a diagnosis of exclusion, although there are inconsistencies in how it is applied, and some pathologists feel that it is not adequately defined. $^{34}$ In a study of the various causes of sudden unexpected death in infancy (SUDI), the most common nonSIDS diagnosis was infection $(7.1 \%$ of 623 cases), followed by cardiovascular anomaly $(2.7 \%)$, child abuse $(2.6 \%)$, and metabolic/genetic disorders $(2.1 \%){ }^{5}$ Cardiovascular defects and serious infection are potentially easier to diagnose at necropsy than many of the inherited metabolic disorders that may contribute to SUDI. Importantly, it was noted that a non-SIDS diagnosis was reached much more frequently in centres with expertise in paediatric pathology $(18 \% \quad v 6 \%) .{ }^{5}$ Therefore, inherited metabolic disorders (IMDs) or other genetic disorders may be underdiagnosed as a cause of SUDI in less specialist centres. IMD as a cause of SUDI will probably constitute a greater proportion of all cases of SUDI as the incidence of total SUDI falls, partly because of a decline in the incidence of other causes of SUDI, and possibly more consistent application of the diagnostic criteria for SUDI; however, an important factor will be improved detection of the various metabolic causes of SUDI.

\section{"Inherited metabolic disorders or other genetic disorders may be underdiagnosed as a cause of sudden unexplained death in infancy in less specialist centres"}

Where SUDI occurs in more than one sibling in the same family, parents may be suspected of maltreatment, and this culture of suspicion has been promoted by some authorities who encourage the consideration of homicide in all cases of SUDI. ${ }^{6}$ It has been suggested that about $10 \%$ of the current annual total of SUDI cases are covert homicide. ${ }^{7}$ Several murder convictions associated with multiple SUDI cases have recently been highlighted in the UK media. An expert witness at the 1999 trial of Sally Clark, who was convicted of murdering her two infant sons, stated that the chance of two children in an affluent nonsmoking family dying of cot death was "one in 73 million". 8 However, the statistical basis for this statement was shown to be seriously flawed and Mrs Clark's conviction was overturned in January 2003. At the subsequent trial of a mother accused of murdering her three small children, the first two deaths having originally been ascribed to SIDS, the possibility of a genetic disorder being responsible for the deaths of these children was presented, and the case ended in acquittal; in a third case, another mother convicted of killing her two baby sons was freed on appeal in December 2003. ${ }^{9}$

The expert opinion for the prosecution in these cases was based on the assumption that individual cases of SUDI occurring in the same family carry independent risk, making the chances of two or three occurring in the same family so small as to be negligible. However, a proportion of cases of SUDI are known to be caused by inherited metabolic or other genetic disorders, many of which are of autosomal recessive inheritance, and thus are associated with a high risk of recurrence in siblings. It has been shown that in families where there has been a diagnosis of SIDS there is an increased risk of further cases, ${ }^{10}$ and it seems plausible that even more heritable causes of SIDS will be uncovered in the future.

In the appeal court judgement in the Clark case, Professor RW Byard, forensic pathologist in Adelaide, Australia, stated that there had been "...significant and ongoing problems in the investigation of these deaths. Standard protocols were not followed and essential steps... were omitted.... Investigations into the pathology and circumstances of sudden infant death are often scanty and inexpert."11

In the UK, it is recommended that the postmortem examination should be conducted by a paediatric pathologist or pathologist with a special interest in paediatric work. ${ }^{12}$ However, paediatric pathology services in the UK are undoubtedly under resourced at present, a situation exacerbated by the paucity of trained paediatric pathologists, limited funding of coroners' investigations in some areas, and issues regarding the retention of human organs; these factors contribute to variability in approaches to the investigation of SUDI. In addition, the lack of a standardised and comprehensive approach to the metabolic investigation is a further important factor in the variability and quality of pathological evidence in the investigation of SUDI.

Most IMDs are the result of a single gene defect, resulting in the deficiency of a specific enzyme, with a widely varying and frequently non-specific clinical presentation. Diagnosis in vivo is often not straightforward, and generally depends upon a series of complex biochemical tests on blood and other tissues. In 1984, a disorder of fatty acid oxidation-medium chain acyl CoA dehydrogenase (MCAD) deficiencywas the first example of an inherited metabolic disorder to be recognised as the cause of death in an infant who had originally been classified as having died of SIDS. ${ }^{13}$ Further cases of MCAD deficiency in "SIDS" cases were subsequently identified, with a notably high incidence of previous unexplained deaths in siblings. Other disorders of fat oxidation, and other inherited metabolic disorders, including organic acidaemias, amino acid defects, and respiratory chain disorders, have all subsequently been implicated in SUDI. However, the complexity of the diagnosis of these disorders, combined with a general lack of expertise and resources to proceed with comprehensive metabolic investigation in cases of SUDI, results in some cases being under investigated. The importance of a protocol for the laboratory investigation of SUDI has been emphasised (MA Preece et al. Archives of Disease in Childhoodabstracts of RCPath Royal College of Paediatric and Child Health Annual Meeting (2001)). ${ }^{14}$

Diagnosing IMD after death is further complicated by the fact that the biochemical determination of enzymatic activity is problematic in postmortem tissue, which is often taken many hours, if not days, after death. Comprehensive guidance is given by Olpin in his review article, ${ }^{15}$ and is also detailed on the Foundation for the Study of Infant 
Death's website. ${ }^{16}$ Both of these reviews highlight that it is crucial to take samples at the earliest possible opportunity, ideally at first presentation to the accident and emergency department, to maximise the chances of making a diagnosis, because the interpretation of biochemical results from tissue and body fluid becomes increasingly difficult as time elapses after death. The coordination of taking and handling these early samples in the accident and emergency department should form part of an agreed protocol into the investigation of all potential SUDI cases.

The advent of tandem mass spectrometry (MS/MS) has made the diagnosis of many inherited metabolic disorders much easier, making it possible to identify many different metabolites simultaneously from dried blood spot specimens and/or bile spots on filter paper. ${ }^{17}$ This relatively new technology is fast, reliable, sensitive, and specific, particularly for the diagnosis of fat oxidation defects, although interpretation in postmortem specimens may be difficult. The combination of this invaluable investigation with a skin biopsy facilitates the confirmation of a diagnosis suggested by MS/MS using enzymatic studies in cultured fibroblasts. ${ }^{18}$ For the optimal targeting of postmortem investigations, which is especially important in the current climate of public feeling towards tissue or organ retention, as much clinical information as is available must be passed on to the pathologist as early as possible; adequate information is very often unavailable at the time of necropsy. ${ }^{3}$

"The advent of tandem mass spectrometry has made the diagnosis of many inherited metabolic disorders much easier"

A definitive diagnosis of the cause of SUDI is obviously highly desirable. This is particularly crucial when there are multiple cases of SUDI in a family, which might indicate an inherited defect, not only to prevent unnecessary and distressing criminal investigation, but also for the purposes of genetic counselling and prenatal diagnosis. If a significant proportion of SUDI cases truly are caused by inherited metabolic disease, then neonatal screening by MS/ MS has the potential to prevent at least some of these deaths. In the meantime, resources should be made available to ensure that all SUDI cases are investigated in centres that have expertise in paediatric histopathology, with access to specialised metabolic biochemical facilities, according to standardised protocols.

J Clin Pathol 2005;58:20-21.

doi: 10.1136/icp.2004.020677

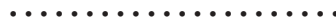

Authors' affiliations

C M Loughrey, Department of Clinical Chemistry, Belfast City Hospital, Lisburn Road, Belfast BT9 7AB, UK

M A Preece, Department of Clinical Chemistry, West Midlands Regional Laboratory for Neonatal Screening and Inherited Metabolic Disorders, Diana, Princess of Wales Hospital, Birmingham B4 6NH, UK A Green, Department of Clinical Chemistry, West Midlands Regional Laboratory for Neonatal, Screening and Inherited Metabolic Disorders

Correspondence to: $\operatorname{Dr} C M$ Loughrey, Department of Clinical Chemistry, Belfast City Hospital, Lisburn Road, Belfast, BT9 7AB, UK ; clodagh.loughrey@bll.n-i.nhs.uk

\section{REFERENCES}

1 Byard RW, Krous HF. Sudden infant death syndrome: overview and update. Pediatr Dev Pathol 2003:6:112-27.

2 Mortality by cause, England and Wales, 2002. London: Office for National Statistics, series DH2, number 29, 2003.

3 Limerick SR, Bacon CJ. Terminology used by pathologists in the reporting on sudden infant deaths. J Clin Pathol 2004;57:309-1 1 .

4 Beckwith JB. Defining the sudden death syndrome. Arch Pediatr Adolesc Med 2003;157:286-90.

5 Cote A, Russo P, Michaud J. Sudden unexpected deaths in infancy: what are the causes? J Pediatr 1999; 135:437-43.

6 Green MA. A practical approach to suspicious death in infancy-a personal view. J Clin Pathol 1998;51:561-3.

7 Levene S, Bacon CJ. Sudden unexpected death and covert homicide in infancy. Arch Dis Child 2004;89:443-7.

8 Dyer C. Sally Clark freed after appeal court quashes her conviction. BMJ 2003;326:304.

9 Dyer O. Meadow faces GMC over evidence given in child death cases. BMJ 2004;328:9.

10 Oren J, Kelly DH, Shannon DC. Familial occurrence of sudden infant death syndrome and apnea of infancy. Pediatrics 1987;80:355.

11 R vs. Sally Clark. Judgment of the Court of Appeal 11 4, 2003.

12 CESDI-executive summary of the 7th annual report, 2000. London: Maternal and Child Health Research Consortium.

13 Howat AJ, Bennet MJ, Shaw L, et al. Mediumchain acylcoenzyme A dehydrogenase deficiency presenting as sudden infant death. BMJ 1985;288:976.

14 Moore A, Debelle G, Symonds KE, et al. Investigation of sudden unexpected deaths in infancy. Arch Dis Child 2000;83:276-9.

15 Olpin SE. The metabolic investigation of sudden infant death. Ann Clin Biochem 2004;41:282-93.

16 Sudden unexpected deaths in infancy: suggested guidelines for accident and emergency departments. London: Foundation for the Study of Infant Deaths (http://www.sids.org.uk/fsid/ pubsdownload.htm).

17 Chace DH, DiPerna JC, Mitchell BL, et al. Electrospray tandem mass spectrometry for analysis of acylcarnitines in dried postmortem blood specimens collected at autopsy from infants with unexplained cause of death. Clin Chem 2001;47:1166-82

18 Green A, Preece MA, Hardy D. More on the metabolic autopsy. Clin Chem 2002;48:964-5 